\title{
NGHIÊN CỨU GIÁ TRI CHỤP CÃT LỚP VI TÍNH ĐA DÃY TRONG CHẨN ĐOÁN LÓC TÁCH ĐỘNG MẠCH CHỦ TẠI BỆNH VIỆN E
}

Vũ Quốc Đôngl , Đỗ Đức Thịnh ${ }^{2}$, Trần Công Hoan ${ }^{3,4}$ Nguyễn Minh Châu $u^{3}$, Nguyễn Trần Thủ $y^{2 *}$

\section{TÓM TẮT}

Muc tiêu: Nghiên cứu nhằm đánh giá một số đặc điểm hình ảnh và giá trị của cắt lớp vi tính đa dãy trong chẩn đoán (LĐMC) có đối chiếu với phẫu thuật và định hướng can thiệp tại Bệnh viện $\mathrm{E}$.

Đối tượng và phương pháp: Phân tích tiến cứu và hồi cứu dữ liệu lâm sàng và các đặc điểm cắt lớp vi tính đa dãy ở 33 bệnh nhân LĐMC chủ tại Trung tâm Tim mạch, Bệnh viện $\mathrm{E}$ từ tháng 2 năm 2020 đến tháng 6 năm 2021.

Kết quả: Trong nghiên cứu có 33 bệnh nhân LĐMC, nhóm tuổi từ 61-70 chiếm nhiều nhất, tuổi trung bình $(62.21 \pm 11.42)$. Bệnh nhân nam chiếm chủ yếu $75,76 \%$. Các triệu chứng lâm sàng và biến chứng nổi bật hơn ở nhóm LĐMC type $\mathrm{A}$, đặc biệt là triệu chứng đau ngực, tăng huyết áp, chèn ép tim cấp. Đặc điểm hình ảnh thường gặp nhất trên cắt lớp vi tính là có vách nội mạc $(96,96 \%)$, đường kính lòng giả >lòng thật chiếm $(96,96 \%)$, dấu hiệu mỏ chim chiếm (96,96\%), ngấm thuốc kém lòng giả chiếm (80\%), và vôi hóa lòng mạch chiếm $(51,51 \%)$. Giá trị chẩn đoán của CLVT đối với lóc tách động mạch chủ type $\mathrm{A}$ có độ nhạy $92,9 \%$ và độ đặc hiệu $100 \%$.

Kết luận: Chụp cắt lớp vi tính đa dãy cho thấy giá trị cao khi mô tả các đặc điểm tổn thương LĐMC và phân biệt type tốt giúp định hướng cho phẫu thuật và can thiệp.
Tù khóa: lóc tách động mạch chủ, chụp cắt lớp vi tính đa dãy, máu tụ trong thành, lồng nội mạc, phẫu thuật Hybrid.

\section{VALUE OF MULTIDETECTOR COMPUTED TOMOGRAPHY IN THE DIAGNOSIS OF AORTIC DISSECTION AT E HOSPITAL}

\section{ABSTRACT}

Objective: The study aims to evaluate some imaging features and value of multidetector computed tomography in the diagnosis of aortic dissection compared with surgery and intervention orientation at $\mathrm{E}$ hospital.

Subjects and methods: Prospective and retrospective analysis of clinical data and multidetector computed tomography features in 33 patients with aortic dissection at Cardiology Center, E Hospital from February 2020 to June 2021.

Results: In the study, there were 33 patients with aortic dissection, the age group from 61-70 accounted for the most, the mean age (62.21 \pm 11.42$)$. Male patients accounted for mainly $75.76 \%$. Clinical symptoms and complications were more prominent in the type A aortic dissection group, especially symptoms of chest pain, hypertension, and acute cardiac tamponade. The most common imaging features on computed tomography are endothelium

\footnotetext{
${ }^{1}$ Khoa Chẩn đoán hình ảnh, Bệnh viện Thu Cúc

${ }^{2}$ Trung tâm Tim mach, Bệnh viện E

${ }^{3}$ Khoa chẩn đoán hình ảnh, Bệnh viện $E$

${ }^{4}$ Bộ môn Chẩn đoán hình ảnh, Truòng Đại học Y Hà Nội

*Tác giả liên hệ: Nguyê̂n Trần Thủy; Email: drtranthuyvd@gmail.com

Ngày nhận bài: 30/08/2021 Ngày cho phép đăng: 30/09/2021
} 
septum (96.96\%), pseudovascular lumen diameter $>$ true lumen $(96.96 \%)$, bird beak sign $(96.96 \%)$, poor contrast enhancement of the false lumen $(80 \%)$, and calcification of the lumen (51.51\%). The diagnostic value of CT for type A aortic dissection has a sensitivity of $92.9 \%$ and a specificity of $100 \%$.

Conclusions: Multidetector computed tomography showed high value when describing the characteristics of aortic dissection lesions and good type discrimination to help guide surgery and intervention.

Key word: aortic dissection, multidetector computer tomography, intramural hematoma, endothelial intussusception, Hybrid surgery.

\section{ĐẠT VẤn Đ Ề}

LĐMC là bệnh lý nguy hiểm, diễn biến cấp tính, xuất hiện do rách lớp áo giữa lan dọc theo động mạch chủ và các nhánh chính. Một số trường hợp ĐMC có thể vỡ ra, máu chảy ồ ạt dẫn tới tử vong. Thường gặp hơn là LĐMC xảy ra khi xuất hiện vết rách nội mạc, dòng máu qua vết rách nội mạc làm lóc tách các lớp áo của động mạch chủ [1]. Tạo ra lòng giả và lòng thật, lóc tách lan dọc đường đi của động mạch, có thể lan xuống bụng, lan vào các mạch tạng, mạch chi gặp nhiều biến chứng nguy hiểm.

Hiện nay nhờ sự phát triển các phương tiện chẩn đoán hình ảnh đặc biệt là máy chụp cắt lớp vi tính thế hệ mới đa lát cắt, bệnh nhân lóc tách động mạch chủ được phát hiện sớm, chẩn đoán nhanh, định hướng được cho phẫu thuật và can thiệp hiệu quả, chính xác.

\section{ĐỐI TƯợNG VÀ PHƯONG PHÁP NGHIÊN CÚU}

Đối tượng: Từ 2/2020 đến 6/2021, 33 bênh nhân chẩn đoán LĐMC đã được phẫu thuật và can thiệp tại Trung tâm Tim mạch, Bệnh viện E.

Kỹ Thuật hình ảnh: Quy trình chụp CLVT với máy chụp CLVT 64 dãy (GE Healthcare, Hoa Kỳ). Thuốc cản Thuốc cản quang Ominipaque 350mgI/ml. Tốc độ tiêm 4$5 \mathrm{ml} / \mathrm{s}$. Toàn bộ hình ảnh được gửi lên hệ thống lưu trữ và truyền hình ảnh (Pacs: carestream, minerva), tái tạo ba mặt phẳng và tái tạo mạch máu theo kĩ thuật MIP.

\section{Phân tích các dấu hiệu trên CLVT}
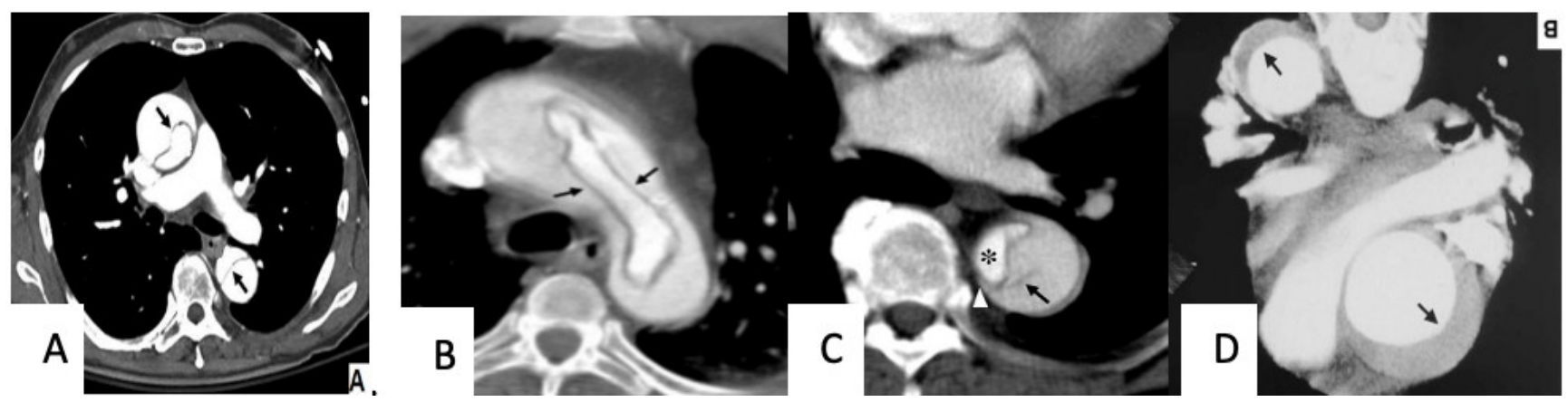

Hinh 1. Dấu hiệu lóc động mạch chủ trên CLVT

Chú thích: A.Tách động mạch chủ kinh điển. B. Hình ảnh vách áo trong. C. Dấu hiệu phân biệt lòng thật - lòng giả: Dấu hiệu mỏ chim (mũi tên trắng); Dấu hiệu mạng nhện (mũi tên đen); Lòng thật (dấu hoa thị) D. Tụ máu trong thành có tiêm thuốc cản quang 
Dấu hiệu vách nội mạc: vách nội mạc giảm tỷ trọng thì trước tiêm, sau tiêm không ngấm thuốc nằm trong lòng động mạch chủ [2]. Dấu hiệu lồng nội mạc với nội mạc: quan sát thấy động mạch chủ trên lát cắt axial, là một đường viền liên tục, lớp áo trong nằm hoàn toàn trong lòng giả quai động mạch chủ gọi là lồng áo trong với áo trong [3]. Dấu hiệu mỏ chim: hình góc nhọn giữa lòng thật và lòng giả do máu tụ nêm vào, thấy trên phim chụp sau tiêm thuốc cản quang tĩnh mạch. Dấu hiệu phân biệt lòng thật lòng giả: nhờ các dấu hiệu dịch chuyển vào trong lòng nội mạc của lớp nội mạc canxi hóa, đường kính lòng giả lớn hơn lòng thật, sau tiêm ngấm thuốc lòng giả kém hơn lòng thật. Dấu hiệu mạng nhện: hình các đường giảm tỷ trọng nằm trong lòng giả trong thì tiêm thuốc cản quang tĩnh mạch [3]. Dấu hiệu máu tụ trong thành: hình tăng tỷ trọng hình liềm ôm quanh động mạch chủ thì trước tiêm thuốc cản quang, sau tiêm không ngấm thuốc [4]. Dấu hiệu loét xo vũua: Ổ đọng thuốc cản quang trong thành động mạch chủ trong vùng

\section{KẾT QUẢ}

máu tụ trong thành ở thì sau tiêm thuốc cản quang [5]. Dấu hiệu loét trong thành động mạch chủ: Ố loét trong thành do mảng xơ vữa bị ăn mòn qua lớp nội mạc đến lớp giữa gây tụ máu trong lớp giữa [6]. Huyết khối trong lòng giả: huyết khối trong lòng giả không ngấm thuốc sau tiêm. Dấu hiệu Mercedes-Ben: hình tách thứ phát trong lòng giả tạo thành 3 lòng [6]. Lóc tách ĐMC kèm phình động mạch chủ: đường kính chỗ sau lớn hơn 1,5 lần đoạn trước liền kề với nó hoặc giá trị bình thường của đường kính ĐMC. Các nhánh tổn thuoong: do LĐMC lan vào các nhánh lan vào ĐM cảnh, ĐM thân tạng, ĐM mạc treo tràng trên, ĐM thận, ĐM chi dưới hai bên gây ra những biến chứng nguy hiểm.

Phuơng pháp xủ lý số liệu: Các số liệu thu thập được trong nghiên cứu được thu thập theo bệnh án được số hóa và xử lý bằng thống kê toán học trong $\mathrm{y}$ học bằng phần mềm SPSS 22.0

Đạo đức nghiên cứu: Nghiên cứu được thông qua theo quyết định số 1768 của trường Đại học Y Hà Nội.

Bảng 1. Đặc điểm chung và lâm sàng của bệnh nhân LĐMC

\begin{tabular}{|c|c|c|c|c|c|}
\hline \multirow{2}{*}{\multicolumn{2}{|c|}{ Biến số }} & \multirow{2}{*}{$\begin{array}{c}\text { Tổng } \\
(n=33)\end{array}$} & \multicolumn{2}{|c|}{ Loại lóc tách ĐMC } & \multirow[b]{2}{*}{$\mathbf{P}$} \\
\hline & & & $\begin{array}{l}\text { Type A } \\
(\mathrm{n}=13)\end{array}$ & $\begin{array}{c}\text { Type B } \\
(n=20)\end{array}$ & \\
\hline \multicolumn{2}{|c|}{ Tuổi (Mean \pm SD) } & $62.21 \pm 11.42$ & $65.62 \pm 8.51$ & $60 \pm 12.68$ & 0,17 \\
\hline \multicolumn{2}{|c|}{ Giới nam } & $25(75,75)$ & $9(69,2)$ & $16(80)$ & 0,48 \\
\hline \multirow{3}{*}{$\begin{array}{l}\text { Lý do } \\
\text { Vàoviện }\end{array}$} & Đau bụng & $13(39,39)$ & $4(30,7)$ & $9(45)$ & 0,41 \\
\hline & Đau ngực & $27(81,81)$ & $13(100)$ & $14(70)$ & 0,029 \\
\hline & Tình cờ & $7(21,21)$ & $1(7,6)$ & $6(30)$ & 0,13 \\
\hline \multicolumn{2}{|c|}{ Đau bụng } & $12(36,36)$ & $3(23,07)$ & $8(40)$ & 0,31 \\
\hline \multicolumn{2}{|c|}{ Đau ngực } & $25(75,75)$ & $13(100)$ & $12(60)$ & 0,009 \\
\hline \multicolumn{2}{|c|}{ Khó thở } & $15(45,45)$ & $8(61,53)$ & $7(35)$ & 0,135 \\
\hline \multicolumn{2}{|c|}{ Chèn ép tim cấp } & $9(27,27)$ & $7(13,8)$ & $2(10)$ & 0,006 \\
\hline \multicolumn{2}{|c|}{ THA } & $31(93,93)$ & $12(92.3)$ & $19(95)$ & 0,75 \\
\hline \multicolumn{2}{|c|}{ Tiếng TT van ĐMC } & $9(27,27)$ & $9(69,23)$ & 0 & 0,0001 \\
\hline \multirow{4}{*}{ Tiềnsử } & THA & $32(96,96)$ & $13(100)$ & $19(95)$ & 0,41 \\
\hline & Bệnh ĐMC & $14(42,42)$ & $8(61,53)$ & $6(30)$ & 0,073 \\
\hline & Marfan & $1(3,03)$ & $1(7,6)$ & 0 & 0,208 \\
\hline & Chấnthương & $3(3,03)$ & 0 & $3(15)$ & 0,143 \\
\hline
\end{tabular}


Nhận xét: Tuổi trung bình là 62.21 \pm 11.42 . Trong đó giới nam chiếm chủ yếu (75.75\%). Triệu chứng đau ngực là thường gặp nhất $(75,75 \%)$. Trong đó các triệu chứng và biến chứng xuất hiện ở typ $\mathrm{A}$ nhiều hơn so với type $\mathrm{B}$ có ý nghĩa thống kê như: Đau ngực, Chèn ép tim cấp, tổn thương ĐMC kèm theo $(\mathrm{p}<0,05)$.

Bảng 2. Các dấu hiệu trên cắt lớp vi tính của L円MC

\begin{tabular}{|c|c|c|c|c|c|}
\hline \multirow{2}{*}{\multicolumn{2}{|c|}{ Thể LĐMC\& Các dấu hiệu }} & \multirow{2}{*}{$\begin{array}{c}\text { Tổng } \\
(n=33)\end{array}$} & \multicolumn{2}{|c|}{ Loại lóc tách ĐMC } & \multirow[b]{2}{*}{$\mathbf{P}$} \\
\hline & & & $\begin{array}{l}\text { Type A } \\
(n=13)\end{array}$ & $\begin{array}{c}\text { Type B } \\
(n=20)\end{array}$ & \\
\hline \multicolumn{2}{|c|}{ LĐMC có vách nội mạc } & $32(96,96)$ & $12(92,30)$ & $20(100)$ & 0,75 \\
\hline \multicolumn{2}{|c|}{ LĐMC thể máu tụ trong thành } & $4(12,12)$ & $4(33,33)$ & 0 & 0,008 \\
\hline \multicolumn{2}{|c|}{ Lồng nội mạc } & $4(12,12)$ & $4(33,33)$ & 0 & 0,008 \\
\hline \multicolumn{2}{|c|}{ Vôi hoá lòng mạch } & $17(51,51)$ & $7(53,84)$ & $10(50)$ & 0,41 \\
\hline \multicolumn{2}{|c|}{ Tăng tỷ trọng hình liềm } & $3(9,09)$ & $2(15,38)$ & $1(5)$ & 0,31 \\
\hline \multicolumn{2}{|c|}{ Ổ loét trong thành } & $7(53,84)$ & $6(46,15)$ & $1(5)$ & 0,005 \\
\hline \multicolumn{2}{|c|}{ Phân biệt lòng thật/giả } & $32(96,96)$ & $12(92,30)$ & $20(100)$ & 0,208 \\
\hline \multicolumn{2}{|c|}{ Ngấm thuốc kém lòng giả } & $24(80)$ & $10(76,9)$ & $14(70)$ & 0,66 \\
\hline \multicolumn{2}{|c|}{ Mỏ chim } & $32(96,96)$ & $12(36,36)$ & $20(60,60)$ & 0,208 \\
\hline \multicolumn{2}{|c|}{ Mạng nhện } & $14(42,42)$ & $9(69,23)$ & $5(25)$ & $\mathbf{0 , 0 1 2}$ \\
\hline \multirow{4}{*}{$\begin{array}{l}\text { Nhánh } \\
\text { lan xa } \\
\text { nhất }\end{array}$} & Động mạch thân tạng & $5(15,15)$ & $4(30,76)$ & $1(5)$ & 0,044 \\
\hline & Động mạch MTTT & $3(9,09)$ & $2(15,38)$ & $1(5)$ & 0,31 \\
\hline & Động mạch thận & $6(18,18)$ & $2(15,38)$ & $4(20)$ & 0,733 \\
\hline & Động mạch chậu & $15(45,45)$ & $5(38,46)$ & $10(50)$ & 0,515 \\
\hline
\end{tabular}

Nhận xét: Dấu hiệu vách nội mạc chiếm phần lớn cả hai type (96.96\%). Dấu hiệu lồng nội mạc và tụ máu trong thành chỉ xuất hiện ở type $\mathrm{A}$ mà không thấy xuất hiện trên $\mathrm{B}$ đều có giá trị $(\mathrm{P}=0,008)$. Dấu hiệu màng nhện, ổ loét trong thành gặp chủ yếu và phần lớn ở type $\mathrm{A}$ với $(\mathrm{P}<0,05)$ có ý nghĩa thống kê. Các dấu hiệu khác đi kèm vôi hóa lòng mạch, tăng tỷ trọng hình liềm, phân biệt lòng thật lòng giả, mỏ chim xuất hiện đồng đều cả hai type không có ý nghĩa thống kê $(\mathrm{P}>0,05)$. LĐMC vị trí lan vào các nhánh ĐM thân tạng của type $\mathrm{A}$ nhiều hơn ở type $\mathrm{B}$, có ý nghĩa thống kê.

Bảng 3. Giá trị chẩn đoán LĐMC theo Stanford của cắt lớp vi tính

\begin{tabular}{|l|l|c|c|c|c|c|}
\hline \multicolumn{2}{|c|}{ Đặc điểm } & \multicolumn{4}{c|}{ Sau phẫu thuật } & p \\
\cline { 3 - 7 } & Stanford A & 13 & $100 \%$ & 0 & 0 & 0,0001 \\
\hline \multirow{2}{*}{ Trước phẫu thuật } & Stanford A & \multicolumn{2}{c|}{ Stanford B } & \\
\cline { 2 - 7 } & Stanford B & 1 & $5 \%$ & 19 & $95 \%$ & \\
\hline
\end{tabular}

Nhận xét: Độ nhạy khi sử dụng hình ảnh chụp cắt lớp vi tính 64 dãy để chẩn đoán lóc động mạch chủ thuộc nhóm Stanford A là 0,929 (92,9\%); độ đặc hiệu là 100\%; tỷ lệ dự đoán dương tính (Stanford A) là $100 \%$ và tỷ lệ dự đoán đúng kết quả âm tính (Stanford $\mathrm{B}$ ) là $95 \%$. Giá trị $\mathrm{P}=0,0001$, có giá trị thống kê. 


\section{BÀN LUẬN}

Trong nghiên cứu của chúng tôi : nhóm tuổi từ 61-70 chiếm số lượng nhiều nhất, (Tuổi trung bình: $62.21 \pm 11.42 ; 31-81$ tuổi) tương tự với như tác giả Page và Hoàng Thị Phương Nhung. Và tác giả Page nghiên cứu trong thời gian 17 năm trên 4428 bệnh nhân cho thấy nam giới chiếm $66,9 \%$ với tuổi trung bình 61,5 [7]. Nghiên cứu của chúng tôi có 24 bệnh nhân nam $(72,7 \%)$ và 9 bệnh nhân nữ $(27,3 \%)$, đồng thuận với hầu hết các tác giả khác giả Vũ Ngọc Tú, Hoàng Thị Phương Nhung[8],[9]. Nam giới tỷ lệ cao hơn nữ giới có thể do hút thuốc lá, uống rượu, béo phì, rối loạn chuyển hoá đường và mỡ máu nên lớp nội mạc dễ rách, dễ bị tổn thương.

Lý do vào viện của bệnh nhân thường gặp nhất là đau ngực $(81,8 \%)$, cao hơn so với Vũ Ngọc Tú ( 70,4 \%). Một số bệnh nhân vào viện vì triệu chứng khác như đau bụng (30,7\%), tương tự của Lujing Zhao, thường do LĐMC lan tới bụng chậu hai bên [14].

Đau ngực là triệu chứng rất quan trọng nhất giúp bác sỹ lâm sàng theo dõi, gợi ý chẩn đoán LĐMC. Trong nghiên cứu của chúng tôi, tỷ lệ bệnh nhân có triệu chứng đau ngực chiếm 75,75 $\%$. Triệu chứng khó thở chiếm 45,45\%, trong đó có $15,2 \%$ chèn ép tim cấp, cao hơn tỷ lệ gặp ở 3,7 $\%$ trong nghiên cứu của Vũ Ngọc Tú [9]. Tiếng thổi tâm thu van ĐMC do hở van ĐMC $(27,27 \%)$ cao hơn so với tác giả Vũ Ngọc Tú $(11,1 \%)$ và tác giả Nguyễn Ngọc Tân (23,3 \%). Hấu hết các triệu chứng nổi bật hơn ở nhóm LĐMC type $\mathrm{A}$. Hội chứng Marfan: trong nghiên cứu có 1 bệnh nhân chiếm 3,0 \% thấp hơn so với Vũ Ngọc Tú (11,1\%), và nghiên cứu của Page (4\%). Bệnh nhân có hội chứng Marfan và tiền sử bệnh động mạch chủ xuất hiện toàn bộ ở LĐMC type $\mathrm{A}$, tiền sử chấn thương gặp thì hay gặp hơn ở type B. Ngoải ra, chúng tôi có 96,96,0 \% tăng huyết áp chiếm tỷ lệ rất cao, cao hơn hầu hết các tác giả khác. Có thể do các bệnh nhân có tiền sử tăng huyết áp nhưng không đi khám thường xuyên, không kiểm soát huyết áp dẫn tới tình trạng LĐMC.

Về đặc điểm của LĐMC trên CLVT: đặc điểm của LĐMC thể kinh điển, trong nghiên cứu có 96,96 \% vách nội mạc có tỷ trọng trước tiêm, sau tiêm không ngấm thuốc cao hơn hẳn các nghiên cứu của khác, dấu hiệu lồng nội mạc ít gặp hơn chiếm 12,12 \%. Dấu hiệu vôi hóa trong lòng động mạch có 17 trường hợp chiếm 51,51\%. Đặc điểm của LĐMC thể máu tụ trong thành và lồng nội mạc: có 4 bệnh nhân, chiếm (12,12\%) có LĐMC type A có kèm với tụ máu trong thành khác với các tác giả khác. Trong nghiên cứu của chúng tôi, dấu hiệu vách nội mạc vôi hóa nằm lệch tâm trong lòng mạch chiếm 39,4\%, phù hợp so với các tác giả LePage ( 26-56\%) [11]. Dấu hiệu mỏ chim: là dấu hiệu phân biệt nổi bật, là một trong dấu hiệu quan trọng nhất quan sát thấy trong mọi trường hợp cấp và mạn tính và được chẩn đoán xác định sau tiêm thuốc. Đây là dấu hiệu điển hình chiếm 96,96\% tương tự với tác giả Le Page 100\% [7]. Dấu hiệu phân biệt lòng thật và lòng giả: trong hầu hết các trường hợp, lòng thật có thể xác định được trên CLVT, bằng sự liên tục của nó với lòng ĐMC trước khi LĐMC. Nghiên cứu chúng tôi góp phần đóng góp phân biệt rõ lòng thật và lòng giả. Theo tác giả LePage lòng giả có kích thước lớn hơn lòng thật là dấu hiệu có ý nghĩa với cả giai đoạn cấp tính và mạn tính. Tác giả cho rằng áp lực của lòng giả luôn lớn hơn lòng thật có xu hướng chèn vào lòng thật. Nghiên cứu của chúng tôi lòng giả lớn hơn lòng 
thật chiếm 96,96\% trường hợp cao hơn so với tác giả LePage [11]. Dấu hiệu màng nhện: là hình ảnh chụp sau tiêm thuốc cản quang chỉ thấy trong lòng giả, dấu hiệu màng nhện có độ đặc hiệu cao nhưng độ nhậy thấp. Nghiên cứu của chúng tôi dấu hiệu này có ở 42,42\% $(\mathrm{P}<0,05)$ có ý nghĩa thống kê. Ổ loét trong thành: chiếm tỷ lệ cao trong nghiên cứu của chúng tôi chiếm 53,84\%, chủ yếu gặp type $\mathrm{A},(\mathrm{P}<0,05)$ có ý nghĩa thống kê. Thể $L Đ M C$ : trong nghiên cứu của chúng tôi thể tụ máu trong thành có 3 bệnh nhân thuộc thể không kinh điển, có hai bệnh nhân thể kinh điển có lóc tách kèm có tụ máu trong thành, LĐMC thể máu tụ trong thành thấp hơn so với Castañer [3]. LĐMC Kèm phình ĐMC: nghiên cứu của chúng tôi, LĐMC có bệnh nhân chiếm 30,30\%, gần giống nghiên cứu của Vũ Ngọc Tú, LĐMC kèm phình chiếm 31,3\%[9]. Phình ĐMC gặp chủ yếu ở LĐMC kinh điển có vách nội mạc ( 40\%), thể máu tụ trong thành ít kèm phình hơn $(5,7 \%)$. Các bệnh nhân trong nghiên cứu của chúng tôi, phình thường gặp ở động mạch chủ lên với type $\mathrm{A}$, ở đoạn $\mathrm{ĐMC}$ xuống với Type $\mathrm{B}$, tương ứng với đoạn LĐMC. Trong nghiên cứu có bệnh nhân LĐMC type $\mathrm{B}$ kèm phình lớn $7,5 \mathrm{~cm}$, được phẫu thuật cấp cứu. Các nhánh lan xa: Do LĐMC lan vào ĐM thận, ĐM thân tạng MTTT, ĐM thận, ĐM chậu. Trong nghiên cứu của chúng tôi LĐMC lan vào ĐM thận gây thiếu máu thận chiếm 15,15\% cao hơn tác giả Vũ Ngọc Tú $(6,4 \%)[9]$. Lan xa tới ĐM chậu trong nghiên cứu của chúng tôi chiếm $45,45 \%$, type $\mathrm{B}$ nhiều hơn chiếm $50 \%$. Nhưng chỉ có 5 trường hợp lan vào ĐM thân tạng chiếm $15,15 \%,(\mathrm{P}=0,044)$ có ý nghĩa thống kê. Các biến chứng tràn dịch màng phổi có 7 trường hợp chiếm 21,21\% ít hơn so với tác giả Lujing Zhao, chiếm (49,5\%) và tràn dịch màng ngoài tim chiếm $9,09 \%$ ngang bằng với tác giả Lujing Zhao [12].

Hình ảnh trên CLVT là cơ sở quan trọng để chẩn đoán LĐMC, dựa vào CLVT LĐMC được phân type theo Stanford và De Bakey và Svensson, trong nghiên cứu của chúng tôi đề cập đến LĐMC phân loại theo Stanford. Đánh giá được độ nhạy khi sử dụng hình ảnh chụp cắt lớp vi tính đa dãy để chẩn đoán lóc động mạch chủ thuộc nhóm Stanford A là 92,9\%, độ đặc hiệu là $100 \%$; tỷ lệ dự đoán dương tính (Stanford A) là $100 \%$ và tỷ lệ dự đoán đúng kết quả âm tính (Stanford $\mathrm{B}$ ) là $95 \%$. Giá trị $(\mathrm{P}=0,0001)$ có giá trị thống kê. Kết quả này khá tương đồng khi đánh giá giá trị ưu điểm của chụp CLVT so với MRI, siêu âm qua thực quản, siêu âm qua thành ngực theo tác giả Raimund Erbel và các cộng sự [16], [17]. Sau khi đã được chẩn đoán dựa trên hình ảnh CLVT 64 dãy theo phân loại Stanford hướng tới điều trị bằng các phương pháp điều trị mà thế giới đang triển khai theo tác giả Dorota Sobczyk [18]. Trong nghiên cứu này chúng tôi đã áp dụng phương pháp phẫu thuật Hybird cho các trường hợp 10 bệnh nhân LĐMC type $A$ và 19 LĐMC type $B$. Đối với những bệnh nhân LĐMC type $\mathrm{B}$ không có nhánh lan vào quai, tùy theo trường hợp có thể làm phẫu thuật hybrid và đặt stengraft đơn thuần [19], [20].

\section{KẾT LUẬN}

LĐMC là một cấp cứu ngoại khoa, có thể gây ra tử vong nếu không được chẩn đoán sớm và chính xác. Các triệu chứng lâm sàng thường cấp tính và rõ rệt hơn ở các bệnh nhân LĐMC type $\mathrm{A}$. Kết quả chụp cắt lớp vi tính có giá trị xác định các tổn thương ĐMC, phân loại type LĐMC giúp chẩn đoán chính xác và đưa ra định hướng quyết định điều trị phẫu thuật và can thiệp tiếp theo. 


\section{TÀI LIỆU THAM KHẢO}

1.HiratzkaLF(2010).

\section{ACCF/AHA/AATS/ACR/ASA/SCA/SCAI.}

Guidelines for the Diagnosis and Management of Patients With Thoracic Aortic Disease. Circulation, 121, e266-e369.

2. Braverman AC (2010). Acute Aortic Dissection - Clinician Update. Circulation, 122, 184-8.

3. Castañer E, Andreu M, Gallardo X, et al (2003). CT in nontraumatic acute thoracic aortic disease: typical and atypical features and complications. RadioGraphics, 23 spec, 93-110.

4. M.A. McMahon và C.A.Squirrell (2010). Multidectector CT of aortic dissection: a pictorial review. Radiolographics, 30 (2), 445-460.

5. Song JK, Kim HS, Song JM, et al (2002). Outcomes of medically treated patients with aortic intramural hematoma. Am J Med, 113, 181-7.

6. M.C.Shiau, M.C. Godoy, P.M. de Groot et al (2010). Thoracic aorta: Acute syndromes. Applied Radiology, 39(1),6.

7 . Pape LA, Awais M, Woznicki EM, et al (2015). Presentation, diagnosis, and outcomes of acute aortic dissection. J Am Coll Cardiol, 66(4), 350-358

8. Hoàng Thị Phương Nhung và Đinh Thu Hương(2012). Nghiên cứu đặc điểm lâm sàng, cận lâm sàng và điều trị nội khoa phình tách động mạch chủ cấp tại bệnh viện Tim mạch Việt Nam từ tháng $1 / 2011$ đến tháng 6/2011, Tạp chí Y hoc Việt Nam, tháng 11- số 1/2012, 12-17.

9. Vũ Ngọc Tú(2016) nghiên cứu đặc điểm bệnh lý và kết quả điều trị phẫu thuật lóc tách động mạch chủ cấp tính loại A-stanfod tại bệnh viện hữu nghị Việt Đức. Luận án tiến sỹ, trường đại học Y Hà Nội.

10. Nguyễn Ngọc Tân(2014). Nghiên cứu đặc điểm lâm sàng, cận lâm sàng và kết quả sớm sau phẫu thuật tách động mạch chủ Stanford A. Luận văn thạc sỹ, truoòng đại học Y Hà Nội.

11. LePage M, Quint LE, Sonnad SS, et al (2001). Aortic dissection: CT features that distinguish true lumen from false lumen. Americal Joural of Roentgenol, 177, 207-211.

12. Clinical features and prognosis of patients with acute aortic dissection in China Lujing Zhao, Yanfen Chai, and Zhigang Li Author information Article notes Copyright and License information Disclaimer

13. Erbel R, Aboyans V, Boileau C, et al. ESC Committee for Practice Guidelines. 2014 ESC Guidelines on the diagnosis and treatment of aortic diseases: Document covering acute and chronic aortic diseases of the thoracic and abdominal aorta of the adult. The Task Force for the Diagnosis and 14 Treatment of Aortic Diseases of the European Society of Cardiology (ESC) [J]. Eur Heart J 2014; 35: 2873-2926. [PubMed] [Google Scholar]

15.Clinical features and prognosis of patients with acute aortic dissection in China Lujing Zhao, Yanfen Chai, and Zhigang Li Author information Article notes Copyright and License information Disclaimer

16. 2014 ESC Guidelines on the diagnosis and treatment of aortic diseases, Raimund Erbel, Victor Aboyans et all. 
17. Diagnostic Testing in Acute Aortic in the treatment of aortic arch aneurysms: Dissection Siobhan Coyle - Tadgh Moriarty - Postoperative and midterm outcomes. The Journal Laura Melody - Damien Ryan Published online: of Thoracic and Cardiovascular Surgery. 19 April 2014 Springer Science+Business Media 2013;145(3):S85-S90.

New York 2014.

18. Echocardiography in Acute Aortic Syndromes Dorota Sobczyk, in New Approaches to Aortic Diseases from Valve to Abdominal Bifurcation, 2018

19. Bavaria J, Vallabhajosyula $\mathrm{P}$, Moeller $\mathrm{P}$, Szeto W, Desai N, Pochettino A. Hybrid approaches doi:10.1016/j.jtcvs.2012.11.044

20. Frank J Criado. A percutaneous technique for preservation of arch branch patency during thoracic endovascular aortic repair (TEVAR): retro- grade catheterization and stenting. J Endovasc Ther. 2007;14(1):54-58 . doi:https:// doi.org/10.1583/06-2010.1. 